\title{
Does Diaphragmatic Breathing Technique Really Improve Its Range of Motion? An Objective Assessment.
}

\section{Ana Rita J. Fernandes ${ }^{1}$, Maria Inês Perez ${ }^{1}$, Ricardo T. Ribeiro ${ }^{2}$, Teresa Tomás ${ }^{1}$}

${ }^{1}$ Lisbon Higher School of Health Technology - Department of Sciences and Technologies of Rehabilitation

${ }^{2}$ Lisbon Higher School of Health Technology - Department of Sciences and Technologies of Radiation and Health Biosignals

\section{Introduction}

It has been highly studied that diaphragmatic breathing technique (DBT) is one of the primary basic Cardiorespiratory Physiotherapy procedures, with strong evidence in a more efficient ventilatory function

According to British Thoracic Society, this technique is described as a tidal volume ventilation, using essentially the diaphragm movement, instead of the chest wall and the accessories muscles ${ }^{(2)}$

Likewise, patients with COPD show a decrease of symptoms, a better diaphragmatic range of motion and a more efficient functional capacity and alveolar ventilation with the continuous practice of DBT.

In the contest of evidence-based practice in physiotherapy there isn't enough studies correlating these effects in healthy subjects.

On the other hand, ultrasound imaging represents a simple, safe and non-invasive direct method to assess the diaphragmatic range of motion $(4,5)$

\section{Objectives}

Based on this, our study had the purpose to quantitatively verify the immediate results of diaphragmatic breathing technique (DBT) on diaphragmatic range of motion in healthy subjects.

\begin{tabular}{l} 
Material and \\
Methods \\
\hline
\end{tabular}

$\checkmark 51$ healthy subjects (10 males; 41 females), the average age being 20 years old and body mass index ranging from 15,6 to $34,9 \mathrm{~km} / \mathrm{m}^{2}$.

$\checkmark$ The inspiration time used in the standard protocol was measured on a pre-test with 12 subjects similar to our sample, resulting in a 3 seconds of inspiration starting from a maximum expiration.

$\checkmark$ To perform DBT, all the subjects were guided by a vocal taping for standardizing the ventilatory pattern.

$\checkmark$ Right hemi-diaphragm range of motion was assessed by M-Mode ultrasound imaging, in a supine position.

Measurements were made before and after the DBT implementation. The technique was always performed by the same physiotherapist, during 15 minutes for each subject. The instruction was based on tactile and auditory stimulation as well as breathing instruction to inhale through the nose and exhale orally.

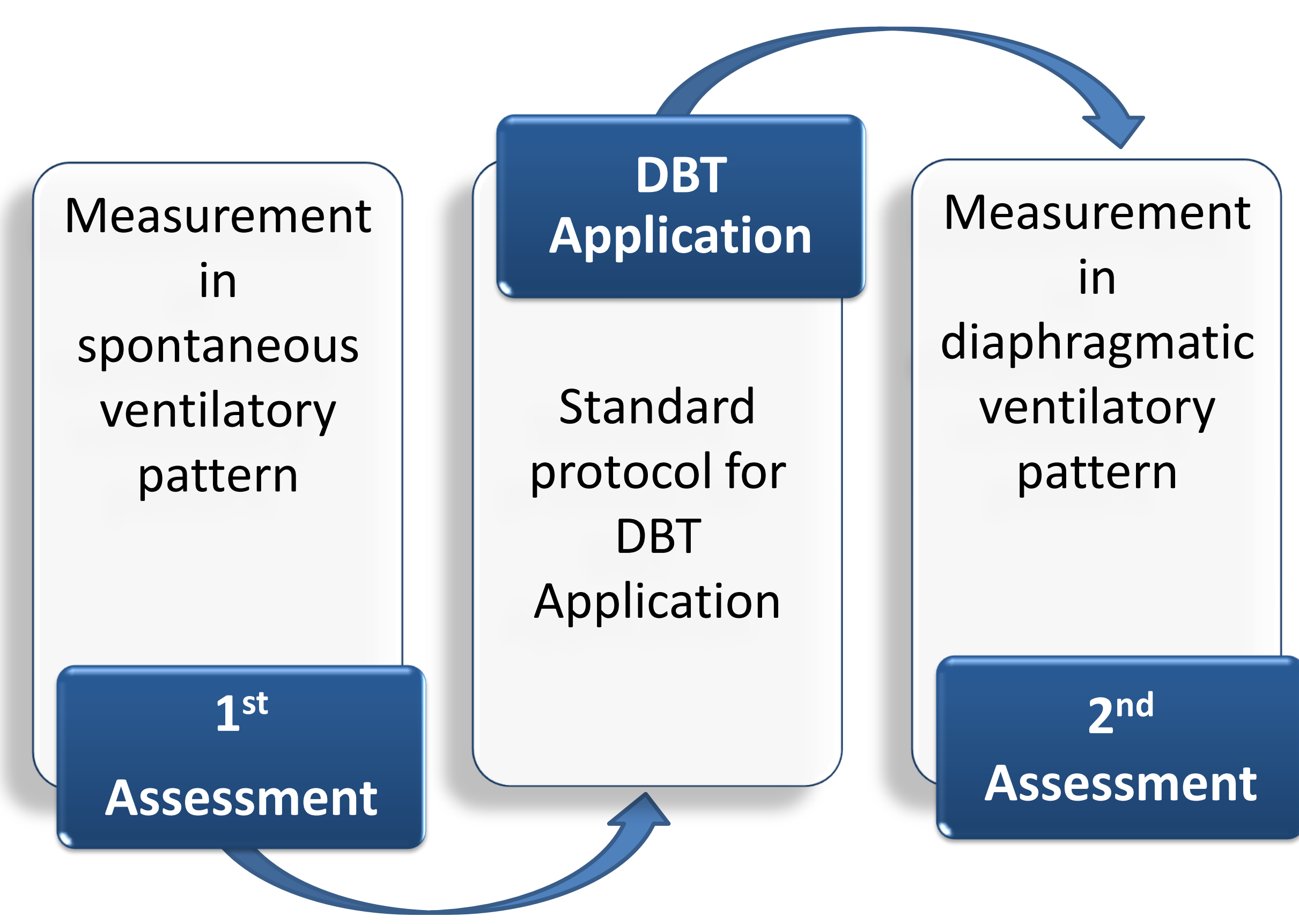

\section{Results}

\begin{tabular}{c|c}
\hline \multicolumn{2}{|c|}{ Right Diaphragmatic Range of Motion } \\
\hline$\frac{1^{\text {st }} \text { Assessment }}{(\text { Before DBT) }}$ & $\frac{2^{\text {nd }} \text { Assessment }}{(\text { After DBT })}$ \\
\hline $55.3 \pm 13,4 \mathrm{~mm}$ & $63.8 \pm 13,2 \mathrm{~mm}$ \\
\hline
\end{tabular}

$\checkmark$ Significant improvement of diaphragmatic range of motion after DBT: $8.5 \pm 14,7 \mathrm{~mm}(p<0.001)$

\begin{tabular}{c|c|c|c|}
\multicolumn{3}{|c|}{$\begin{array}{c}\text { Right Diaphragmatic Range of Motion and Limit } \\
\text { Values in Men and Women }\end{array}$} \\
\hline \multicolumn{3}{|c|}{$1^{\text {st }}$ Assessment } & \multicolumn{2}{|c|}{$2^{\text {nd }}$ Assessment } \\
(Before DBT) & \multicolumn{2}{c|}{ (After DBT) } \\
\hline Male, mm & Female, mm & Male, mm & Female, mm \\
$51,5 \pm 13,9$ & $56,2 \pm 13,3$ & $61,2 \pm 15,7$ & $64,4 \pm 12,7$ \\
$(30-71)$ & $(34-84)$ & $(37-95)$ & $(40-90)$ \\
\hline
\end{tabular}

$\square$ In both assessments, the measurements showed that diaphragmatic range of motion was greater in women than in men.

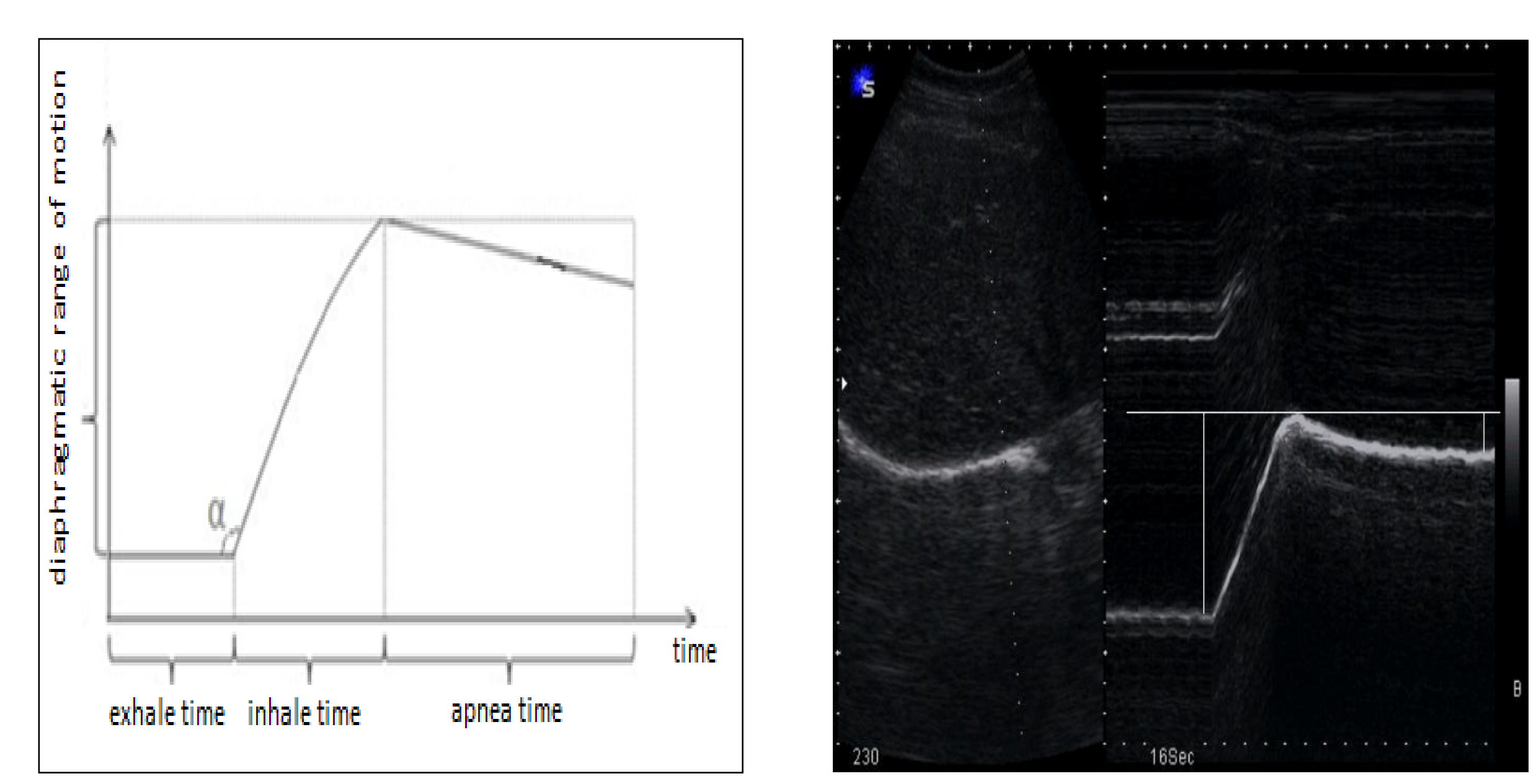

A strong correlation between the slope and the diaphragmatic range of motion after DBT was found $(r$ $=-0.71, p<0.001)$

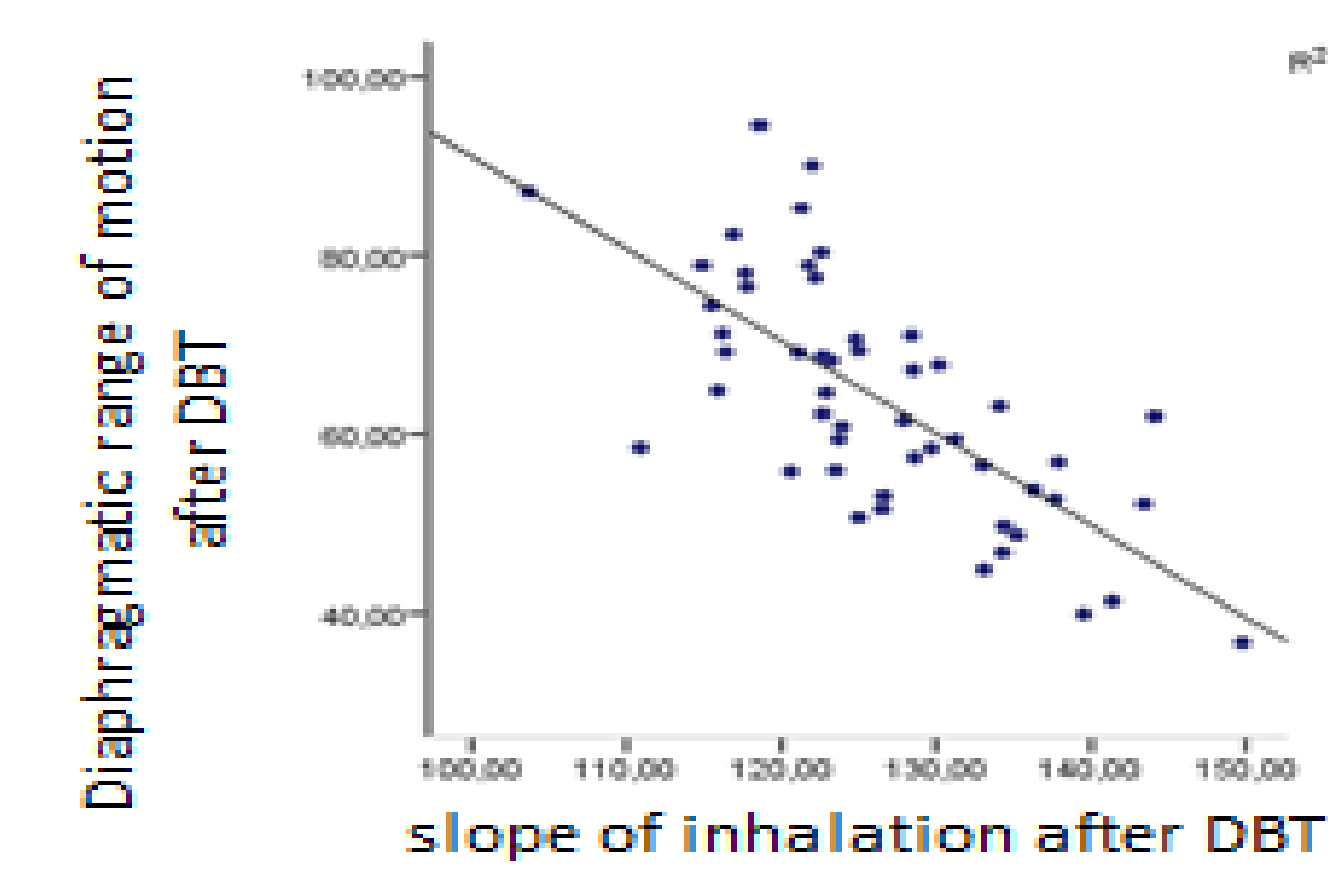

\section{Conclusions and Clinical Implications}

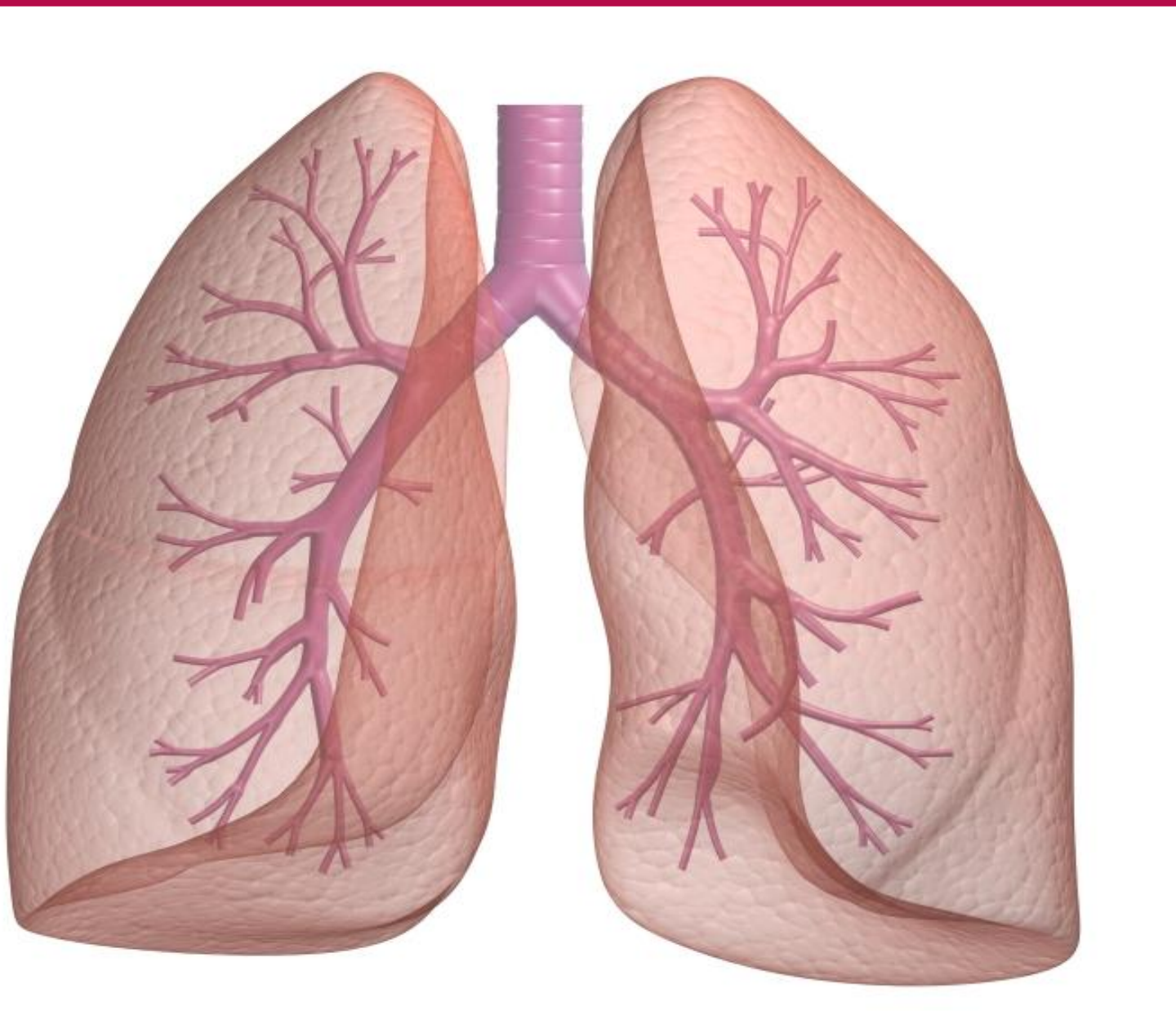

To our knowledge this is the first study to assess the effects of DBT on range of motion of diaphragm muscle with ultrasound imaging i Based on ultrasound measurements, it has been proved that DBT really contributes to a higher diaphragmatic range of motion. Future! jstudies are needed in order to understand the influence of protocol parameters (e.g. inspiration time).

In the contest of evidence-based practice in physiotherapy, it has been showed by objective measurements that DBT improves the ¡diaphragm range of motion, translating into a more efficient ventilatory function and thus can be used in clinical setting.

Contacts Mobile Phone: (+351) 918418283 Email: rita.jsf hotmail.com Maria Inês Perez
Ma Teresa Tomás

Mobile Phone: (+351) 962528947 Email: teresa.tomas@estesl.ipl.pt 